\title{
A COMPARATIVE HISTOPATHOLOGICAL STUDY OF EPITHELIAL LININGS OF ODONTOGENIC CYSTS AND UNICYSTIC AMELOBLASTOMAS
}

\begin{abstract}
J Sudiono, RB Zain. A Comparative Histopathological Study of Epithelial Linings of Odontogenic Cysts and Unicystic Ameloblastomas, Annals Dent Univ Malaya 1998; 5: $29-34$
\end{abstract}

\begin{abstract}
The epithelial cystic linings and adjacent connective tissues of 61 cases of odontogenic cysts (radicular cysts[RC], dentigerous cysts[DC] and odontogenic keratocysts[OKC]) and unicystic ameloblastomas(UA) were described and compared histopathologically. The type of epithelium in relation to the presence of rete processes and the distribution of chronic inflammatory cells were analyzed statistically. Significant associations between the presence of rete processes in the non-keratinized epithelial linings and inflammation in the subjacent connective tissues of $\mathrm{RC}$ and $\mathrm{DC}$ were found in this study. There was also a statistically significant association between the presence of rete Processes and nonkeratinized epithelial linings in $\mathrm{OKC}$. The results also showed that in inflamed OKC, the cystic lining epithelium exhibited hyperplasia indistinguishable from lining epithelium of RC and DC. This study further showed that ameloblastomatous-like epithelial cystic linings were present in inflammed odontogenic cysts. All except for one case of unicystic ameloblastomas in this study showed ameloblastomatous epithelial cystic linings. It is recommended that the lining epithelium of RC and DC be examined carefully in order to rule out OKC. Similarly, ameloblastomatous-like lining epithelium arising from chronic inflammation in $\mathrm{RC}$ and DC should be differentiated from true ameloblastomatous cystic lining. Such careful examinations are diagnostically important in view of the similarities of epithelial cystic linings of inflamed $\mathrm{OKC}$ with DC and RC aggressive behavior of OKC and UA.
\end{abstract}

Keywords: odontogenic cyst, unicystic ameloblastoma, histopathological features

\section{INTRODUCTION}

The epithelial linings of radicular cysts (RC), dentigerous cysts (DC) and odontogenic keratocysts(OKC) histologically may appear similar in areas of inflammation(1). Some of these cysts such as the dentigerous cysts may have hyperplastic epithelium, which may appear as ameloblastomatous-like proliferations. Rests of odontogenic epithelium may also be present in odontogenic cysts which could be mistaken for ameloblastoma and salivary gland tumours(2). Since 1925, many had reported the development of ameloblastoma within the walls of odontogenic cysts and the most commonly cited was the dentigerous cyst (3-7). In 1977, Robinson and Martinez used the term 'unicystic ameloblastoma'(UA) for such lesions(3). The term unicystic ameloblastoma was later used in many reports and is now a generally accepted termi-
1 Janti Sudiono, ${ }^{2}$ Rosnah Binti Zain

1 Lecturer

Department of Pathology, Anatomy, Faculty of Dentistry, Trisakti University, Indonesia

2 Associate Professor

Department of Oral Pathology, Oral Medicine and Periodontology

Dental Faculty, University of Malaya, Malaysia

nology. Unicystic ameloblastoma describes a unilocular ameloblastoma that has a single cystic space in which there is intraluminal or mural growth. This terminology may also represent an odontogenic cyst in which there has been ameloblastic transformation of the epithelial lining (8)

The odontogenic keratocyst linings in inflamed areas may present as a non-keratinized stratified squamous epithelium, which may appear similar to RC and DC. Such similarities in the appearance of odontogenic cyst linings (RC, DC and $\mathrm{OKC}$ ) associated with inflamed areas would pose a diagnostic problem for odontogenic keratocyst as it maybe mistaken for dentigerous cysts. Such misdiagnoses should be avoided since the biologic activities of OKC differed from those of radicular or dentigerous cysts. Most radicular and dentigerous cysts grow slowly and do not attain a large size. However, the OKC shows a more aggressive biologic behaviour where a high recurrence rate was observed in $\mathrm{OKC}$ as compared to radicular and dentigerous cysts $(9,10)$. Similarly, the appearance of ameloblastomatous-like epithelial linings in inflamed radicular or dentigerous cysts may lead to overdiagnosis of unicystic ameloblastomas. Unicystic ameloblastoma has a better prognosis; conventional ameloblastoma. However, the mural type of UA where ameloblastomatous islands of epithelium have perforated the cyst wall, may behave aggressively similar to a conventional ameloblastoma(15). Thus, it is very important to distinguish UA from $\mathrm{RC}$ and $\mathrm{DC}$ as UA requires a close followup.

In view of the similar histopathological features and the presence of ameloblastomatous-like epithelial linings observed in different types of odontogenic cysts in areas of inflammation, it is the purpose of this paper to describe and compare the histopathological features of cyst linings of odontogenic cysts (RC, DC and $\mathrm{OKC}$ ) and unicystic ameloblastomas.

\section{MATERIALS AND METHODS \\ Materials}

A total of 61 cases of odontogenic cysts and unicystic ameloblastomas $(\mathrm{RC}=18 ; \mathrm{DC}=16 ; \mathrm{OKC}=15 ; \mathrm{UA}=12$ ) was 
studied. These specimens had been fixed in $10 \%$ buffered formalin and had been embedded in paraffin wax. Four micrometer $(\mathrm{pm})$ paraffin sections of selected specimens were stained with haematoxylin and eosin. The specimens used were biopsy materials from the Department of Oral Pathology, Oral Medicine and Periodontology, Faculty of Dentistry, University of Malaya.

\section{Criteria}

The criteria used for odontogenic cysts were according to the WHO classifications in 1992(1) where a radicular cyst is defined as a cyst arising from the epithelial residues (rests of Malassez) in the periodontal ligament as a consequence of inflammation, usually following the death of the dental pulp. The dentigerous cyst is defined as a cyst, which encloses the crown and is attached to the neck of an unerupted tooth and develops by accumulation of fluid between the reduced enamel epithelium and the crown. or between the layers of the reduced enamel epithelium. The odontogenic keratocyst is defined as a cyst arising in the tooth bearing areas of the jaws, or posterior to the mandibular third molar, and characterised by a thin fibrous capsule and a lining of keratinized stratified squamous epithelium usually about five to eight cells in thickness and generally without rete ridges.

The criteria for unicystic ameloblastoma is an ameloblastoma which radiographically presents as a unilocular radioluscent lesion mimicking odontogenic cyst and had developed in the wall of an odontogenic cyst or had extended into the lumen of the cyst or involve only the cystic lining $(1,3,8)$.

\section{Methodology}

Clinical characteristics of the patients were obtained from the information written by clinicians in the biopsy forms. The age of the patients, ethnicity and gender distribution according to the type of lesions were recorded.

The histopathological features of odontogenic cysts and unicystic ameloblastomas were noted. Evaluation for the type of epithelium in relation to the presence of rete processes and the distribution of chronic inflammatory cells within the subjacent connective tissue wall was carried out for odontogenic cysts. The correlation between the types of epithelium, presence of rete processes and distribution of inflammation in the subjacent connective tissue of these lesions were analyzed statistically using Kolmogorov
Smirnov test. The significant association was decided if the value of $\mathrm{D}$ maximum was higher than the critical value at 0.05 level.

\section{RESULTS}

\section{Clinical characteristics}

The mean age of all the 61 patients with lesions in this study was $32.75 \pm 15.34$ years. For patients with $\mathrm{RC}, \mathrm{DC}, \mathrm{OKC}$, and UA, the mean ages were $33 \pm 14.54,35.5 \pm 17.97,31.6 \pm 16.75$, and $30.17 \pm 11.79$ years respectively. Their range of ages were 14 to 68 years for RC, 8 to 74 years for DC, 12 to 67 years for $\mathrm{OKC}$, and 16 to 47 years for UA.

For all lesions, there was almost an equal distribution in men and women with men to women ratio of $1: 1.03$. There was a higher predilection for women for $\mathrm{RC}, \mathrm{OKC}$, and $\mathrm{UA}$ while there was a higher predilection for men for DC (Figure 1).

Out of 61 cases of odontogenic cysts and UA, 41 cases (67\%) occurred in Chinese. The prevalence in Chinese was $61 \%(11 / 18)$ for RA, 63\%(10/16) for DC, 67\%(10/15) for OKC and $83 \%(10 / 12)$ for UA. There was no OKC in Malays and no UA in Indians in this sample.

\section{Histopathological features}

Table 1 shows the distribution of lesions according to the types of epithelial cystic linings. Out of a total of 18 cases of RC, 16 cases ( $89 \%$ ) were lined by a non-keratinized stratified squamous epithelium while 2 cases were predominantly lined by a ciliated pseudostratified squamous epithelium with small foci of non-keratinized stratified squamous epithe- 


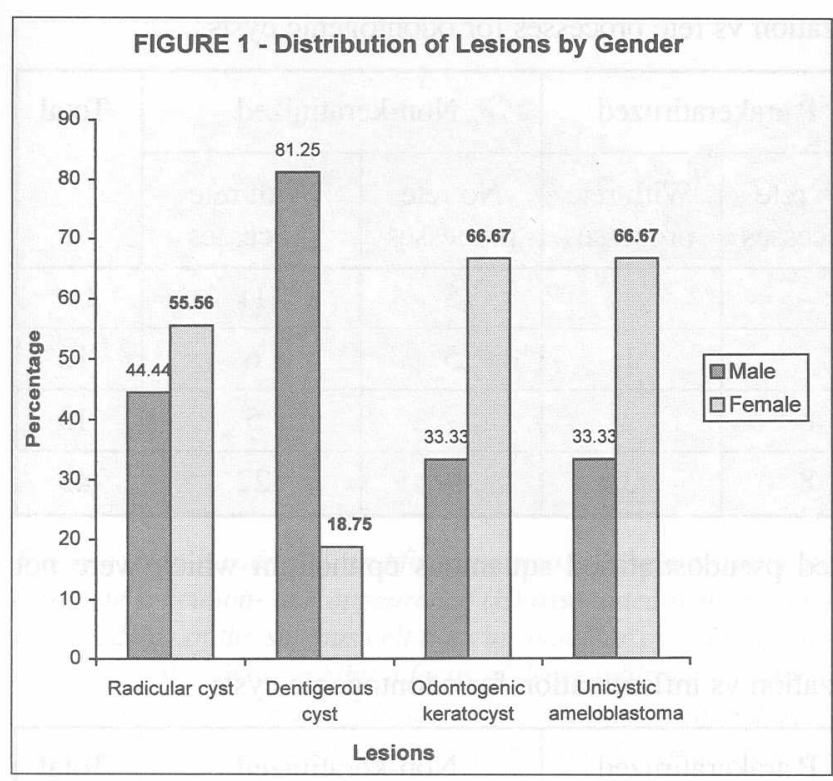

Figure 1: The distribution of odontogenic cysts and unicystic ameloblastoma according to gender

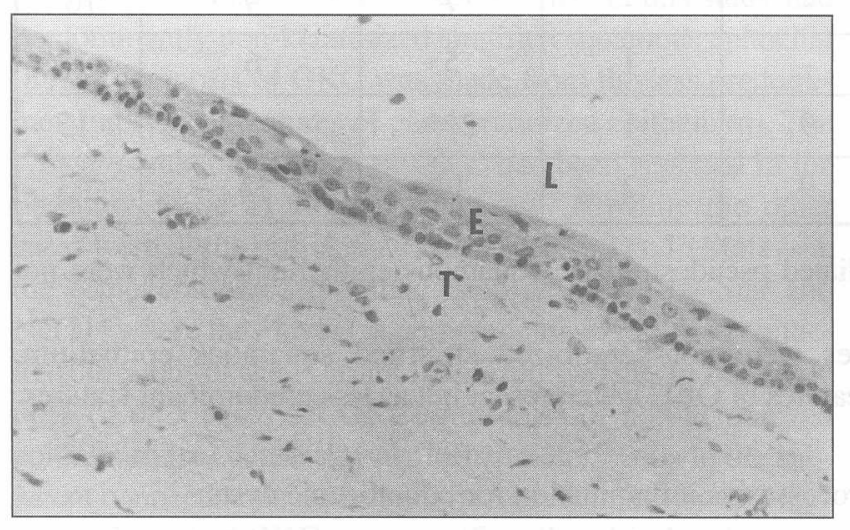

Figure 2: Thin non-keratinized stratified squamous epithelium with no rete processes in dentigerous cyst. (Haematoxylin and Eosin stain; Original magnification - 50X). $L=$ lumen; $E=$ cyst lining epithelium; $T=$ connective tissue in cyst wall.

lial linings. For DC, 11 out of 16 cases $(68.8 \%)$ were lined by a non-keratinized stratified squamous epithelium (Figure 2) while 5 cases showed other varieties of epithelium. All cases of OKC in this study had parakeratinized epithelium (Figure 3). Approximately 33.3\% (5 of 15 cases) of these parakeratinized epithelium showed the mixtures of pare and non-keratinized stratified squamous epithelium with a predominance of non-keratinized epithelium. All cases of UA showed a non-keratinized stratified squamous epithelial linings.

Eleven out of 16 cases of RC(69\%) and 6 out of 11 cases of $\mathrm{DC}(55 \%)$ with non-keratinized epithelial lining showed irregular epithelial downgrowth where in some the rete processes formed arcades(Figure 4). For OKC, 80\%(8/10) of cases with parakeratinized epithelium showed no rete processes while in all 5 cases where there are predominant areas of non-keratinized stratified squamous epithelium, there were rete processes associated with such epithelium

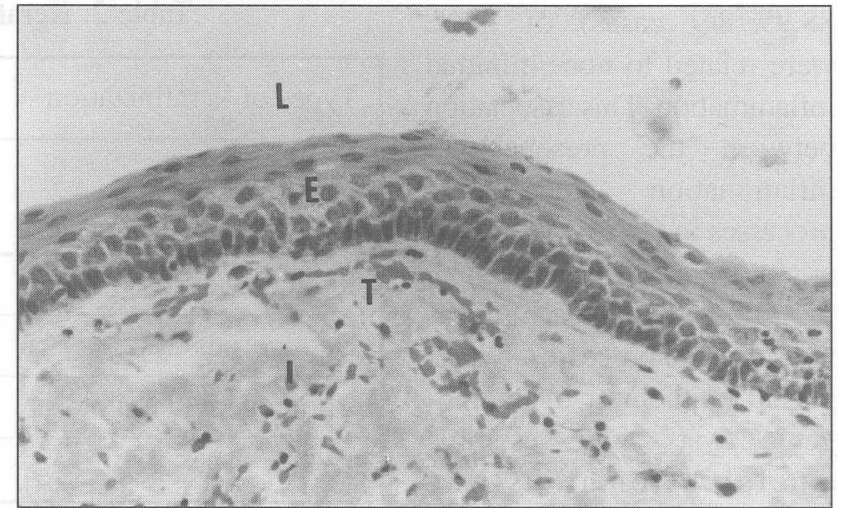

Figure 3: Thin parakeratinized stratified squamous epithelium with pallisading of basal cell nuclei in odontogenic keratocyst. No or minimal inflammation in the cyst wall. (Haematoxylin and Eosin stain; Original magnification - 50X). $L=$ lumen; $E=$ cyst lining epithelium; $T$ = connective tissue in cyst wall.

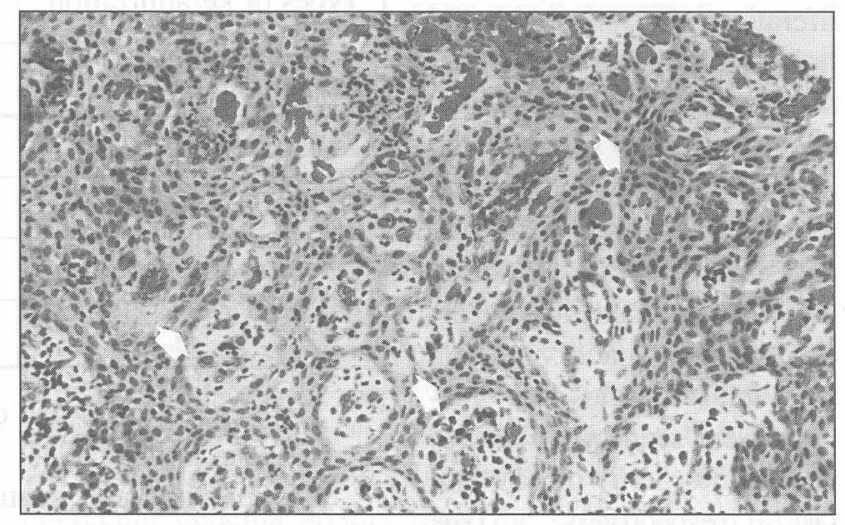

Figure 4: Radicular cyst showing epithelial proliferation into irregular rete processes forming arcades (white arrowheads). The subjacent connective tissue contains an abundance of inflammatory cells. (Haematoxylin and Eosin stain; Original magnification - 1 OOX). $L=$ lumen; $T$ = connective tissue in cyst wall.

(Table 2). The association between the presence of rete processes and non-keratinized epithelial linings in OKC was found to be statistically significant $(\mathrm{D}=0.71>0.70)$.

Table 3 shows $56 \%$ (9/16) of RC, 53\%(6/11) of DC and $60 \%(3 / 5)$ of OKC with predominantly non-keratinized stratified squamous epithelial cyst linings were associated with dense distribution of chronic inflammatory cells in the subjacent connective tissue. However, these associations between non-keratinized stratified squamous epithelial cyst lining and the presence of chronic inflammation were not statistically significant.

Table 4 shows that $90 \%(8 / 9)$ of RC and all cases of DC with the rete processes were related to areas of dense chronic inflammation in the subjacent connective tissue. The association between the presence of rete processes and dense chronic inflammation in the subjacent connective tissue was found to be statistically significant for $R C(D=0.78>0.64)$ and DC (D - 1>0.68). For OKC, $50 \%$ of cases with rete processes were related to dense chronic inflammation and the other $50 \%$ to none/minimal inflammation in the subjacent connective tissue. Among the cases with no rete processes 
$88.9 \%$ (8/9 cases) of OKC were related to none/minimal inflammation. This association between the presence of inflammation and rete processes in OKC was however, not significant.

Other than presenting as irregular rete processes, hyperplastic epithelium in odontogenic cysts may resemble ameloblastomatous-like epithelium when associated with heavily inflammed subjacent connective tissue(Figure 5). The latter epithelial feature in areas of inflammation seemed to arise from the proliferation of cystic epithelial lining into the underlying connective tissue with intercellular oedema in the spinous cell area of cyst epithelium leading to a stellate reticulum-like appearance while the basal layer maintained its hyperchromatic nuclei. Such ameloblastomatous-like features were seen in 50\%(9/18) and $12.5 \%(2 / 16)$ of $\mathrm{RC}$ and DC respectively. True ameloblastomatous epithelial cyst linings (Figure 6) were observed in all UA except for 1 case. The diagnosis of UA was made for this single case without ameloblastomatous lining based on the presence of ameloblastomatous islands within the cyst wall.

\section{DISCUSSION}

The predominance of non-keratinized stratified squamous epithelium in RC and DC in this study was in keeping with many reports in the literature $(7,10)$. Two cases

of $\mathrm{RC}$ in this study had ciliated pseudostratified squamous epithelium. This type of epithelium with mucous metaplasia is usually seen as microscopic variations of the radicular cyst epithelium (8). Among these non-keratinized epithelial linings in RC, 1 case (5.5\%) also showed an area of localized parakeratinization. Localized keratinization in $\mathrm{RC}$ was also observed by Browne (7) where he reported its presence in $2 \%$ of radicular cysts. Keratinization was seen in 1 out of 16 cases of $\mathrm{DC}(6.3 \%)$ in this study. According to Browne (7), keratin metaplasia may occur in dentigerous cyst leading to mainly included in this table. included in this table.

\begin{tabular}{|l|c|c|c|c|c|}
\hline Types of keratinization & \multicolumn{2}{|c|}{ P arakeratiruzed } & \multicolumn{2}{c|}{ Non-keratinized } & \multirow{2}{*}{ Total } \\
\hline Rete processes & $\begin{array}{c}\text { No rete } \\
\text { processes }\end{array}$ & $\begin{array}{c}\text { With rete } \\
\text { processes }\end{array}$ & $\begin{array}{c}\text { No rete } \\
\text { processes }\end{array}$ & $\begin{array}{c}\text { With rete } \\
\text { processes }\end{array}$ & \\
\hline Radicular cyst & - & - & 5 & 11 & $16^{*}$ \\
\hline Dentigerous cyst & - & 1 & 5 & 6 & 12 \\
\hline Odontogenic keratocyst & 8 & 2 & - & 5 & 15 \\
\hline Total & 8 & 3 & 10 & 22 & 43 \\
\hline
\end{tabular}

* The other 2 cases had ciliated pseudostratified squamous epithelium which were not

Table 3: Keratinization vs inflammation for odontogenic cysts

\begin{tabular}{|l|c|c|c|c|c|}
\hline \multirow{2}{*}{ Types of keratinization } & \multicolumn{2}{|c|}{ P arakeratiruzed } & \multicolumn{2}{c|}{ Non-keratinized } & \multirow{2}{*}{ Total } \\
\cline { 1 - 5 } Inflammation & Non/light & Dense & Non/light & Dense & \\
\hline Radicular cyst & - & - & 7 & 9 & $16^{*}$ \\
\hline Dentigerous cyst & - & 1 & 5 & 6 & 12 \\
\hline Odontogenic keratocyst & 9 & 1 & $2 * *$ & 3 & 15 \\
\hline Total & 9 & 2 & 14 & 18 & 43 \\
\hline
\end{tabular}

* The other 2 cases had ciliated pseudostratified squamous epithelium which were not

** The predominant features were non-keratinized stratified squamous epithelium. However the diagnostic features of OKC were present but as less predominant features.

Table 4: Rete processes vs inflammation for odontogenic cysts

\begin{tabular}{|l|c|c|c|c|c|}
\hline \multirow{2}{*}{ Rete processes } & \multicolumn{2}{|c|}{ No rete processes } & \multicolumn{2}{c|}{ With rete processes } & \multirow{2}{*}{ Total } \\
\cline { 1 - 5 } Inflammation & Non/light & Dense & Non/light & Dense & \\
\hline Radicular cyst & 8 & 1 & 1 & 8 & 18 \\
\hline Dentigerous cyst & 9 & - & - & 7 & 16 \\
\hline Odontogenic keratocyst & 8 & 1 & $3+$ & $3+$ & 15 \\
\hline Total & 25 & 2 & 4 & 18 & 49 \\
\hline
\end{tabular}

+ These 6 cases showed predominant features of irregular rete processes. Diagnostic features of $\mathrm{OKC}$ exhibiting no rete processes were observed as less predominant features.

orthokeratinization of a part of the cyst lining. However, keratinizing metaplastic cases must be differentiated from an odontogenic keratocyst lining (8). This study had shown parakeratin formation instead of orthokeratin in one case of dentigerous cyst. In this case the diagnosis of odontogenic keratocyst was excluded since typical features of hyperchromatic and pallisading of basal cell nuclei were absent. Browne(7) also reported the presence of mucous metaplasia in $40 \%$ of DC. His result was higher than that found in the present study(18.8\%). 


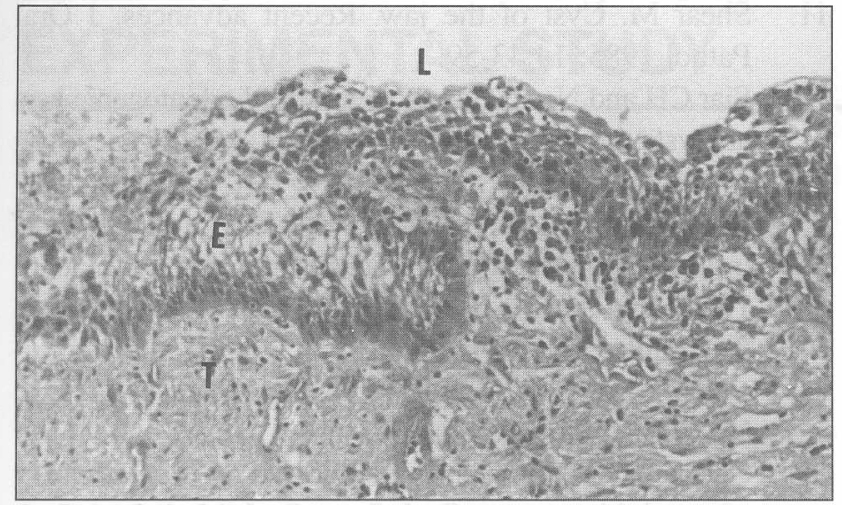

Figure 5: Non-keratinized stratified squamous epithelium exhibiting a stellate reticulum-like appearance (E) associated with intercellular oedema of the spinous cell area in radicular cyst. The subjacent connective tissue exhibit a focus of dense infiltrate of chronic inflammatory cells. (Haematoxyllin and Eosin stain; Original magnification - 100X). $L=$ lumen; $E=$ epithelium; $T=$ connective tissue in cyst wall.

All cases of OKC in this study were of the parakeratinized type. Only 5 out 15 cases of OKC in this study had predominantly non-keratinized stratified squamous epithelial lining (diagnosis of OKC was made from the less predominant but typical features of parakeratinized epithelium). The typical parakeratinization of $\mathrm{OKC}$ had been reported in the literature to be in $90-95 \%$ of $\operatorname{cases}(7,10)$ while the other cases were of the orthokeratinized type $(7,9,11)$. In Malaysia, the orthokeratinized type had been described by Siar and $\mathrm{Ng}(11)$ where they had also observed non-keratinized epithelium in 2 out of the 9 orthokeratinized variant. In our study $60 \%$ ( 3 of 5 cases) of OKC with areas of non-keratinized epithelium had dense chronic inflammatory cells in the subjacent connective tissue and all the 5 predominantly non-keratinized cases of $\mathrm{OKC}$ were significantly related to the presence of rete processes. Such changes of epithelial lining of OKC from parakeratinized to non-keratinized type in relation to the presence of rete processes and inflammation within the OKC in this study was also described in other reports $(7,9,13,14)$. Rodu et al (13) reported that $78 \%$ of 112 OKC exhibited marked inflammation and that this is associated to a significant degree with the transformation of the accompanying cyst lining to a non-keratinized form seen routinely in inflammatory odontogenic cysts. Similar observation was reported by Kakarantza et al (14) where $72.4 \%$ of 87 cases of OKC showed inflammation with transformation into nonkeratinized epithelial lining. The transformation of epithelial lining into non-keratinized type may also influence a change in the biologic behaviour of $\mathrm{OKC}$ to a less aggressive lesion(14).

Hyperplastic cystic epithelial linings usually in relation to inflammation of cyst wall in many cases exhibit rete process formation. Inflammed OKC usually exhibit similar characteristics of hyperplastic cystic epithelial linings of RC and DC. This result supported previous reports that hyperplastic epithelium are often seen in areas of inflammation in $\mathrm{RC}(7,8)$ and such epithelial hyperplasia leading to rete process formation may also be noted in areas of inflamma-

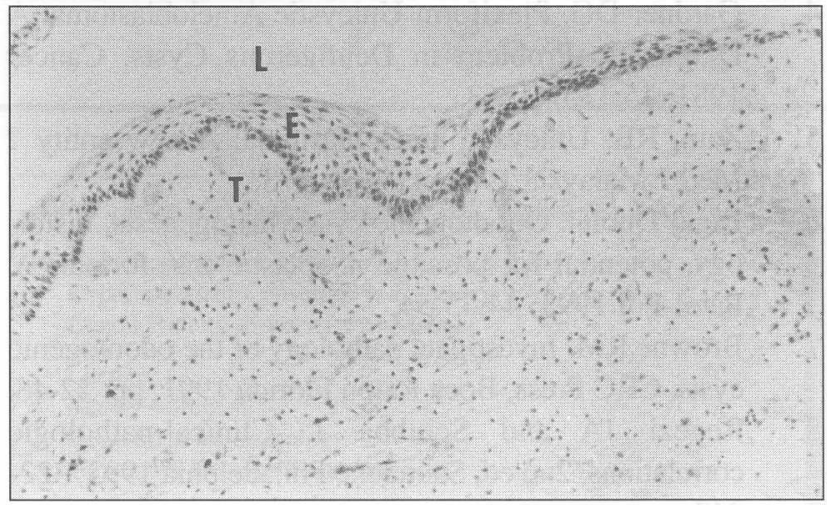

Figure 6: Unicystic ameloblastoma with ameloblastomatous epithelial lining. (Haematoxylin and Eosin stain; Original magnification - 50X). $L=$ lumen; $E=$ epithelium; $T=$ connective tissue in cyst wall.

tion or secondary infection of DC cyst linings(7). In view of the more aggressive behavior of $\mathrm{OKC}$ as compared to $\mathrm{RC}$ and $\mathrm{DC}$, it is recommended that for diagnostic purposes, careful examination of most of the RC and DC cystic linings is required to rule out the possibility of an inflamed $\mathrm{OKC}$ which is similar to inflamed RC and DC.

Hyperplastic epithelium may also resemble ameloblastomatous lining epithelium in RC and DC as observed in this study. Such observations may raise the possibility of ameloblastoma(14). However, since this type of feature was also associated with a dense inflammatory cell infiltrate where the stellate-reticulum like epithelium was a result of intercellar oedema arising from the presence of chronic inflammation in the area, it should be considered as not diagnostic of unicystic ameloblastoma. In view of the reported ameloblastic potential of DC (15), it is thus important to be able to recognise true ameloblastomatous epithelium from ameloblastomatomatous - like epithelium. In most cases of odontogenic cysts, the presence of ameloblastomatous-like epithelial lining in inflammed odontogenic cysts is insufficient to diagnose unicystic ameloblastoma unless other more diagnostic features of unicystic ameloblastoma are evident. However, such features in the absence of subepithelial inflammation can be diagnostic of unicystic ameloblastoma. Otherwise, differentiation between ameloblastomatous and ameloblastomatous-like lining epithelium can be difficult and therefore additional criteria for UA as described by Robinson and Martinez(3)and Gardner(15) should be applied.

\section{REFERENCES}

1. Kramer IRH, Pindborg JJ, Shear M. Histological typing of odontogenic tumours. 2ne ed, Springer- Verlag, Berlin 1992; pp 34-42,100-109.

2. Gardner DG. A Pathologist's approach to the treatment of ameloblastoma. J Oral Maxillofac Surg 1984; 42:161-166.

3. Robinson L and Martinez MG. Unicystic ameloblastoma. A prognostically distinct entity. Cancer 1977; 40:278-285. 
4. Gardner DG. Plexiform Unicystic Ameloblastoma: A Diagnostic Problem in Dentigerous Cysts. Cancer 1981; 15:1359-1363.

5. Zain, RB. Unicystic ameloblastoma: A new entity ? Med J Malaysia 1986; 41 :370-373.

6. Bigaj Dubiel. Odontogenic cysts: histogenesis, histology, potential for possible neoplastic transformation. Patol-Pol 1993; 44:45-54.

7. Browne RM. Investigate pathology of the odontogenic cysts. CRC Press, Boca Raton Florida 1991; pp: 22-48.

8. Regezi JA and Sciubba J. Clinical-pathologic correlations, 2nd ed, Saunders, Philadelphia 1993; 322 343.

9. Anand VK, Arrowood JP, Krolls SO. Odontogenic keratocysts: a study of 50 patients. Laryngoscope 1995; 105(1):14-16.

10. Yeo JF, Zain RB, Zhao YY. Clinico pathologic study of 259 cases of radicular cysts in Singapore and Malaysia Asian J Oral Maxillofac Surg 1996 ; 8: 135-144.
11. Shear M. Cyst of the jaw. Recent advances. J Oral Pathol 1985; 14:43-59.

12. Siar $\mathrm{CH}$ and $\mathrm{Ng} \mathrm{KH}$. Orthokeratinized odontogenic keratocysts in Malaysians. Br J Oral Maxillofac Surg 1988; 26:215-220.

13. Rodu B, Tate AL, Martinez M. The implication of inflammation in odontogenic keratocyst. J Oral Pathol 1987; 16:518-521.

14. Kakarantza AK, Nicolatou O. Odontogenic keratocysts: Clinicopathologic study of 87 cases. J Oral Maxillofac Surg 1990; 4:593-599.

15. Gardner DG. Some current concepts on the pathology of ameloblastomas. Oral Surg Oral Med Oral Path 1996; 82:660-669.

\section{ACKNOWLEDGEMENTS}

The authors would like to thank Mr Koh Lam Seng and the Oral Pathology General Staff for their assistance in preparing the study materials. 